\title{
Atomic Study on Defects in 2D PtSe 2 Monolayers Using Electron Microscopy
}

Jun Chen ${ }^{1}$ and Jamie Warner ${ }^{2}$

${ }^{1}$ Department of Materials, University of Oxford, Oxford, England, United Kingdom, ${ }^{2}$ Walker Department of Mechanical Engineering, The University of Texas at Austin, Austin, Texas, United States

Two-dimensional (2D) transition metal dichalcogenides (TMDs) are layered semiconductors with unique electronic and optical properties which have shown immense potential in ultrathin (opto-) electronic devices. ${ }^{1}$ Structural defects that are ubiquitous in 2D materials have demonstrated to exert significant impacts on the materials' properties. In the past several decades, 2D TMDs of group VIB metal $\mathrm{MX}_{2}$, like $\mathrm{M}=\mathrm{Mo}, \mathrm{W}$, and $\mathrm{X}=\mathrm{S}$, Se, typically with hexagonal $2 \mathrm{H}$ polytype, have been studied intensively in fundamental structures, properties, and applications. They possess sizable band gaps in the range of 1.5$2.0 \mathrm{eV},{ }^{2}$ corresponding to the red to near infrared regions. While the wider range of optoelectronic applications require further exploration of novel 2D materials for full cover of the colour regions. The emerging noble metal TMDs, e.g. $\mathrm{PtX}_{2}$, have recently gained a lot of attention for expanding the 2D family, due to their fascinating properties such as the layer-controllable transition from metal to semiconductor, the notable carrier mobility, strong interlayer interaction, anisotropy, and ultrahigh air stability. ${ }^{3-8}$ The tunable band gaps in the range of $0.25-1.6 \mathrm{eV}$ of $\mathrm{PtX}_{2}$ make up the gap between graphene and most other TMDs, enabling their promising utilization in the desirable mid-infrared photonics and optoelectronics. ${ }^{9-12}$ Differing from the common $2 \mathrm{H}$-phase structures of Mo/W-based TMDs, the $2 \mathrm{D}$ PtX 2 crystal is preferably constructed in 1T polytype. Accordingly, their structural properties are supposed to be differentiated from those in $2 \mathrm{H}$-TMDs. Understanding different defect structures in $1 \mathrm{~T}-\mathrm{PtX} \mathrm{X}_{2}$ and their behaviours is vital for taking full advantage of them in tailoring materials properties. Theoretical studies have predicted the structures and stability of point defects in $1 \mathrm{~T}$-phase $\mathrm{PtX}_{2}$, as well as the affected electronic and magnetic properties, ${ }^{4}, 13-15$ while the experimental research addressing the defects in monolayer $\mathrm{PtX}_{2}$ is still very limited so far, which is crucial for exploiting the great potential of $\mathrm{PtX}_{2}$ for extensive device applications.

In this work, atomically thin $\mathrm{PtSe}_{2}$ films were grown using direct selenization method of ultrathin Pt layer at the atmospheric pressure in a chemical vapour deposition furnace. By using annular dark-field scanning transmission electron microscopy (ADF-STEM) direct imaging with single-atom resolution, we studied detailed atomic structures of vacancies, dislocations and $\mathrm{GBs}$ in 1T-phase 2D $\mathrm{PtSe}_{2}$, which are distinguished from the those defective structures in other hexagonal crystals like graphene, h-BN and $2 \mathrm{H}-$ phase TMDs (e.g. $\left.\mathrm{MoS}_{2}\right)$. The isolated Se vacancies $\left(\mathrm{V}_{\mathrm{Se}}\right)$ are highly mobile under beam irradiation, whose collective behaviours contribute to the re-arrangement of the $\mathrm{PtSe}_{2}$ lattice. Distinct 1D deviated defects are found in $1 \mathrm{~T}-\mathrm{PtSe}_{2}$ exhibiting $1 \mathrm{~T}$ features without losing Se atom rows, which is distinguished from the typical line defects missing rows of chalcogens in 2H-phase TMDs. The main difference lies in the mechanism that in the $\mathrm{PtSe}_{2}$ the increased concentration of $\mathrm{V}_{\mathrm{Se}}$ in a confined $\mathrm{PtSe}_{2}$ grain alters strain fields locally, triggering the formation of a special type of linearly stretched defect with contracted lattice distortion. For the GB structures, a special tilt angle of $30^{\circ}$ is found where the lattice in armchair direction is stitched with that in the zig-zag direction, which is the most frequently occurred GB angle in polycrystalline $\mathrm{PtSe}_{2}$ films. Other GBs with different tilt angles are also directly observed in meandering pathways with various edge dislocation structures assembled, which show distinct 1T-phase structural feature. Taking PtSe 2 as an example, this systematic study of 1T-phase TMDs is expected to enrich our knowledge of defective structures in 2D binary lattices. 
References

1. Manzeli, S.; Ovchinnikov, D.; Pasquier, D.; Yazyev, O. V.; Kis, A. Nat. Rev. Mater. 2017, 2, 17033.

2. Duan, X.; Wang, C.; Pan, A.; Yu, R.; Duan, X. Chem. Soc. Rev. 2015, 44, (24), 8859-8876.

3. Sajjad, M.; Singh, N.; Schwingenschlögl, U. Appl. Phys. Lett. 2018, 112, (4), 043101.

4. Kandemir, A.; Akbali, B.; Kahraman, Z.; Badalov, S. V.; Ozcan, M.; Iyikanat, F.; Sahin, H. Semicond. Sci. Technol. 2018, 33, (8), 085002.

5. Zhao, Y.; Qiao, J.; Yu, Z.; Yu, P.; Xu, K.; Lau, S. P.; Zhou, W.; Liu, Z.; Wang, X.; Ji, W.; Chai, Y. Adv. Mater. 2017, 29, (5).

6. Yao, W.; Wang, E.; Huang, H.; Deng, K.; Yan, M.; Zhang, K.; Miyamoto, K.; Okuda, T.; Li, L.; Wang, Y.; Gao, H.; Liu, C.; Duan, W.; Zhou, S. Nat. Commun. 2017, 8, (1), 14216.

7. Zhao, Y.; Qiao, J.; Yu, P.; Hu, Z.; Lin, Z.; Lau, S. P.; Liu, Z.; Ji, W.; Chai, Y. Adv. Mater. 2016, 28, (12), 2399-2407.

8. Miró, P.; Ghorbani-Asl, M.; Heine, T. Angew. Chem. Int. Ed. 2014, 53, (11), 3015-3018.

9. Yu, X.; Yu, P.; Wu, D.; Singh, B.; Zeng, Q.; Lin, H.; Zhou, W.; Lin, J.; Suenaga, K.; Liu, Z.; Wang, Q.

J. Nat. Commun. 2018, 9, (1), 1545.

10. Pi, L.; Li, L.; Liu, K.; Zhang, Q.; Li, H.; Zhai, T. Adv. Funct. Mater. 0, (0), 1904932.

11. Wang, Z.; Li, Q.; Besenbacher, F.; Dong, M. Adv. Mater. 2016, 28, (46), 10224-10229.

12. Wang, Z.; Wang, P.; Wang, F.; Ye, J. F.; He, T.; Wu, F.; Peng, M.; Wu, P. S.; Chen, Y. F.; Zhong, F.; Xie, R. Z.; Cui, Z. Z.; Shen, L.; Zhang, Q. H.; Gu, L.; Luo, M.; Wang, Y.; Chen, H. W.; Zhou, P.; Pan, A. L.; Zhou, X. H.; Zhang, L. L.; Hu, W. D. Adv. Funct. Mater. 2020, 30, (5), 1907945.

13. Gao, J.; Cheng, Y.; Tian, T.; Hu, X.; Zeng, K.; Zhang, G.; Zhang, Y.-W. ACS Omega 2017, 2, (12), 8640-8648.

14. Yong, X.; Zhang, J.; Ma, X. International Journal of Hydrogen Energy 2020, 45, (15), 8549-8557. 15. Avsar, A.; Cheon, C.-Y.; Pizzochero, M.; Tripathi, M.; Ciarrocchi, A.; Yazyev, O. V.; Kis, A. Nat. Commun. 2020, 11, (1), 4806. 